\title{
Auto-fiction(s) du réel : La Pudeur ou l'Impudeur d'Hervé Guibert
}

\author{
Catherine Guéneau \\ Paris III Sorbonne Nouvelle
}

Je veux qu'on m'y voie en ma façon simple, naturelle et ordinaire, sans contention et artifice : car c'est moi que je peins. [...] je t'assure que je m'y fusse très volontiers peint tout entier, et tout nu.

Montaigne (Les Essais)

Dimanche 22 juillet, dix heures trente, avec le masseur j'ai commencé à expérimenter la vidéo. Je ne me fais plus photographier depuis deux ans, et je ne me suis jamais laissé prendre nu [...], j'ai toujours refusé. Là, je suis nu entre les mains du masseur, et ça filme. [...] j'ai cherché un bon angle en posant le caméscope sur son pied, j'ai appuyé sur le bouton rouge, vérifié qu'il y avait bien « record » dans le viseur, et suis allé m'étendre à plat ventre sur la table de massage, la tête tournée vers la bibliothèque, donc cachée à l'objectif.

Guibert (1991a, p. 98) 
Quand filmeur et filmé ne font plus qu'un, la mise en scène de soi devient centrale et, avec elle, la nécessité de repenser l'intégralité du dispositif filmique. Si l'autobiographie en littérature ou l'autoportrait en peinture et en photographie ont été couramment pratiqués, le cinéma semblait moins adapté techniquement à ce type de réalisation intimiste. L'apparition progressive de dispositifs de filmage plus légers donnera finalement aux cinéastes toute la liberté nécessaire pour se filmer eux-mêmes.

Le seul film d'Hervé Guibert, La Pudeur ou l'Impudeur (1991), reprend ce principe de l'auto-filmage. « Moi, j'écris sans trop de regrets, mais j'enrage du cinéma. » (Guibert, 2001, p. 183) Après avoir échoué au concours de l'IDHEC, il ne parviendra jamais à faire aboutir ses projets de films, jusqu'au jour où Pascale Breugnot, productrice des célèbres émissions de Reality show comme Sexy folies, L'Amour en danger et Perdu de vue dans les années 1980-1990, après l'avoir remarqué sur le plateau d'Apostrophes, lui demande de réaliser un film pour TF1. Le dispositif proposé par la production établit les règles du jeu : muni d'une caméra vidéo amateur et d'un pied, Guibert devra livrer aux spectateurs le quotidien de sa maladie, à savoir le sida. La durée du tournage va s'étendre sur un peu plus de six mois (entre juin 1990 et mars 1991), peu de temps avant son décès en décembre 1991. La trajectoire de Guibert trouvera ainsi une voie imprévisible pour assouvir ce désir de cinéma qui ne l'aura jamais quitté, comme en témoigne cette phrase troublante et prémonitoire, écrite plusieurs années auparavant dans son journal : «Si la mort ne survient pas, naturellement, à son extrémité, il faudra que mon corps, que toute mon énergie s'ébranle dans un projet de cinéma, sinon mon désir de cinéma 
n'aura été qu'un désir de mort [...].» (1991a, p. 183) Ce qui aurait pu ou dû verser dans le pathos et dans les dérives voyeuristes, si caractéristiques des émissions de reality show, aboutira finalement, grâce à la sensibilité artistique de Guibert et à celle de sa monteuse, Maureen Mazurek, à une véritable création filmique, un " cinéma du subjectif » (Leblanc, 2003), au croisement entre enjeux de vie et enjeux de représentation. Un cinéma qui semble vouloir brouiller les frontières entre le cinéma et la vie, entre professionnel et amateur, entre les genres, ni documentaire ni fiction, mais dans un entre-deux, celui peut-être de l'essai cinématographique. Un cinéma des subjectivités, plus ou moins directement exprimées à travers les interactions entre filmeur(s), filmé(s) et spectateur(s). Expression subjective directe, relayée dans ce cas précis, par la médiation d'une caméra. En choisissant l'auto-filmage, Guibert accepte de s'exposer, de se peindre nu, sans intermédiaire, comme dans cette citation des Essais de Montaigne qu'il affectionnait tout particulièrement: «J'ai été très frappé par l'introduction des Essais de Montaigne, qui disait “J'ai voulu me peindre nu", ça a fait tilt, je me suis dit que c'était quelque chose que je pourrais mettre en exergue à tout ce que j'ai fait [...]. » (1992b, p. 145)

«Aller au bout du dévoilement de soi » Guibert résume ainsi son rapport à l'écriture (1990a, p. 247). Le passage pour la première fois de sa vie à l'écriture filmique va lui permettre sans doute de réaliser encore plus intensément et radicalement ce désir. "Quand je disparaîtrai j'aurai tout dit, je me serai acharné à réduire cette distance entre les vérités de l'expérience et de l'écriture. » (1988). L'expérience, ici, prend une forme extrême, face au spectre de la mort, tout comme le corps ne parvient plus à effacer les stigmates de la maladie qui 
le ronge. L'écriture de l'image, elle aussi, déborde de cette présence obsédante. Comme pour mieux retenir un corps qui lui échappe jour après jour, la caméra enregistre mécaniquement cette lente désincarnation. Première scène du film après le générique: Guibert en plan fixe assis, une infirmière lui fait une prise de sang — voix off :

Le processus de détérioration amorcé dans mon sang par le SIDA se poursuit de jour en jour. Bien avant la certitude de ma maladie sanctionné par les analyses, j'ai senti tout à coup mon sang découvert, mis à nu, comme si un vêtement l'avait toujours protégé sans que j'en ai conscience, il me fallait vivre désormais avec ce sang dénudé, exposé, à toute heure dans les transports publics, dans la rue quand je marche, toujours guetté par une flèche qui me vise à chaque instant. Est-ce que ça se voit dans les yeux ? ${ }^{1}$

«Le dévoilement de soi » va se faire littéralement, crûment, par la mise à nu de ce corps qu'on peut dévoiler sans pudeur parce qu'il n'a justement plus rien d'érotique ou de sexuel, un corps livré au médical, à la froideur clinique, surexposé sous les lumières trop vives du champ opératoire ou bien encore noyé sous des lumières jaunâtres de salles d'attente d'hôpitaux, un corps qui n'aura bientôt plus d'autre identité que celle du sida.

«Ce corps décharné [...] je le retrouvais chaque matin en panoramique auschwitzien dans le grand miroir de la salle de bains. » (1991a, p. 15) Filmer au quotidien face à la caméramiroir, comme une lutte éperdue contre la mort, contre la montre aussi, pour tenter de retenir les ultimes lambeaux de vie. Éclairs fugitifs de vitalité, comme ce match de boxe échangé avec la mort, coup de poing face caméra :

${ }^{1}$ Extrait de la bande son du film La Pudeur ou l'Impudeur, texte repris (en partie) d'À l'ami qui ne m'a pas sauvé la vie, p. 13-14. 
J'ai senti la mort venir dans le miroir bien avant qu'elle y ait vraiment pris position. Il n'y avait pas de jour où je ne découvrais une nouvelle ligne inquiétante, une nouvelle absence de chair sur la charpente. Cela avait commencé par une ligne transversale sur les joues et maintenant l'os semblait sortir hors de la peau, à fleur de peau comme de petites îles plates sur la mer. Cette confrontation tous les matins avec ma nudité dans la glace était une expérience fondamentale. (extrait de la bande son du film)

Expérience que Guibert tient à partager intimement avec le spectateur, nu devant la glace, nu dans la baignoire, sur le siège des toilettes, sur la table de massage, nu éprouvant de la difficulté à se vêtir, nu s'immergeant dans la mer. «Ma nudité dans la vidéo est d'ordre pictural et documentaire pas exhibitionniste. » (2001, p. 530), écrit-il dans son journal. Le cadre ne semble pas parvenir à contenir ce corps devenu exagérément grand de par sa maigreur et rendu si maladroit par son extrême faiblesse. "Hier T. m'a emmené à la plage à l'heure où il n'y a plus personne et où le soleil est parti (je ne le supporte plus) et j'ai filmé ma baignade. T. m'a dit que je ressemblais à un grand oiseau de mer, à un pélican. Il a donc compris ce que je fais avec ce film.» (2001, p. 532) Présence obsédante d'un corps rendu si pesant, qui se rappelle à vous sans cesse, à travers chacun des gestes les plus anodins du quotidien, devenus soudain insurmontables : «l'usage même de mes jambes, de mes cuisses, de mes fesses pour me relever d'un siège devenait plus incertain» (2001, p.14). En témoigne encore cette scène pathétique où Guibert, cadré en gros plan pendant la consultation avec son médecin, ne parvient à enfiler sa veste qu'au prix de multiples contorsions.

Comme si tout pouvait soudain se résorber à travers l'histoire de ce corps: «J'ai eu l'impression, par la force des 
choses, d'être mon propre personnage, mais aussi d'être un corps mis en jeu dans des narrations, dans des situations, dans des rapports [...]. » (1992b, p. 145) Se filmer soi-même entraîne inévitablement un effet de distanciation: comme dans toute démarche autobiographique, on assiste à la mise en œuvre d'une dialectique entre soi et le «personnage » construit par le récit, dialectique incessante entre réel et imaginaire. Guibert se raconte tout autant qu'il est raconté par son film.

\section{Interactions vie/cinéma}

L'art et la vie sont inextricablement liés dans l'écriture de Guibert, qu'elle soit filmique ou littéraire, ou encore photographique, mais c'est peut-être dans cet ultime et unique film que cet entrelacement pourra atteindre son paroxysme. Écriture pour échapper à l'angoisse de la mort qui se fait de plus en plus pressante devant l'avancée de la maladie : «Écrire dans le noir ? Écrire jusqu'au bout? En finir pour ne pas arriver à la peur de la mort. » (Guibert, 1992a, p. 93) Le tournage du film et la rédaction du Protocole compassionnel s'entrecroisent sans cesse. On retrouve des fragments de texte en voix off dans le film; quelques passages du livre évoquent directement le tournage, ou bien certaines scènes sont présentes à la fois dans le livre et dans le film. Jusqu'à quel point ces deux écritures de soi se nourrissent-elles l'une de l'autre? Dans les deux cas, Guibert privilégie la méthode du récit intime à la première personne à travers l'écriture d'un journal, journal écrit, duquel il va puiser la matière du livre, journal filmé, qui fournira le matériau de montage. Avec toujours cette même volonté de rapprocher le plus intensément possible l'expérience vécue de sa reconstruction fictive par l'écriture du livre ou du film. «Le 
sida m'a permis de radicaliser un peu plus encore certains systèmes de narration, de rapport à la vérité, de mise en jeu de moi-même au delà même de ce que je pensais possible.» (1990b)

Si Guibert contrôle entièrement le processus du littéraire — mis à part l'imprévisible lié à l'écriture elle-même : "Pas de livre sans structure inattendue dessinée par les aléas de l'écriture. » (Guibert, 1991a, p. 20) —, c'est loin d'être le cas pour le film, qui nécessite des moyens techniques plus difficiles à mettre en œuvre pour lui. Guibert, comme il l'avoue lui même, est «nul avec les appareils»; il ne maîtrise pas du tout la technique : «Je ne savais pas si j'enregistrais vraiment [...] je ne savais pas s'il y avait le son, je ne savais pas s'il y avait le son et pas l'image, ou l'image et pas le son, je ne savais rien et l'idée de voir m'épouvantais. » (1992b) Les conditions de tournage sont difficiles : Guibert est seul, il ne connaît pas les outils et, de plus, il se trouve considérablement affaibli par la maladie. Il ne participera pas non plus véritablement au montage du film, la monteuse, Maureen Mazurek, se trouvant la plupart du temps seule face aux 26 cassettes vidéo enregistrées. Elle jouera d'ailleurs un rôle essentiel dans l'orientation et la structuration du film et Guibert reconnaîtra la «main d'artiste $»^{2}$ qui a su créer une œuvre cinématographique du «fouillis inextricable » et du « casse-tête » angoissant des rushes. Elle a en particulier détourné le projet de départ, où il était question d'y introduire un personnage de fiction, "une voyeuse», comme elle l'a convaincu de positionner la scène du suicide en partie centrale du film, et non pas à la fin, comme Guibert l'avait initialement

2 Voir la dédicace de Guibert à M. Mazurek reproduite dans Artières et Cugnon. 
prévu. Avec une grande humilité, il reconnaîtra cette part de création collective propre au cinéma en déclarant au cours d'un entretien journalistique: "Même si j'en ai tourné toutes les images, je m'en sens moins l'auteur que la productrice et la monteuse. » (1991b) Le film va échapper à son auteur, à l'image de sa propre vie: "Nous sommes entrés dans la zone de l'incontrôlable. », dit-il à propos de sa maladie. " On arrive un stade de la maladie où l'on a plus prise sur elle, il serait vain de croire qu'on peut en maîtriser les mouvements. Je fais un looping en chute libre sur la main du destin. » (1991a, p. 27-28)

Ce qui appartient véritablement à Guibert, ce sont ces fragments de vie enregistrés mécaniquement par la caméra, des situations qu'il a délibérément choisies de mettre en scène parmi toutes celles qui s'offraient à lui dans son quotidien : il écartera par exemple systématiquement tous ses amis proches (à l'exception d'un bref plan où l'on aperçoit son ami T. le portant jusqu'à la mer pour la baignade); seules ses deux grand-tantes, âgées de 85 et 95 ans, seront filmées plus longuement, sans doute parce qu'elles incarnent si bien à elles deux la pulsion de mort et de vie mêlées à la fois. La tentation de la mort et du suicide comme l'irrépressible élan de vie, cette dualité profonde ressentie quotidiennement par l'auteur. Hervé partage avec Suzanne, la plus âgée des deux grand-tantes, cette proximité de la mort à travers ce vieillissement prématuré du corps :

Le SIDA m'a fait accomplir un voyage dans le temps. Un corps de vieillard décharné, affaibli a pris possession de mon corps d'homme de 35 ans. J'ai 95 ans comme ma grand-tante Suzanne qui est impotente. Chaque jour je perds un geste que j'étais encore capable de produire la veille. Je lutte contre la montre. (extrait de la bande son du film) 
Il la questionne: "T'as envie de vivre ou t'as envie de mourir?». Elle répond: " Ça dépend des moments...». En réalité, c'est à lui même que Guibert pose ces questions, tout comme la réponse lui est familière. Lui aussi oscille sans cesse entre la tentation du suicide et celle de lui résister. C'est alors qu'il décide de mettre en scène un vrai-faux suicide devant la caméra. Difficile alors de ne pas faire le rapprochement avec cette phrase, écrite bien avant dans La Mort propagande : «Me donner la mort sur scène, devant les caméras. Donner ce spectacle extrême, excessif de mon corps, de ma mort. » (1977, p. 184). Ce sera sans doute la scène du film où la mise en jeu de l'auteur sera la plus intense, la plus imprévisible. « Toujours à portée de la main: la digitaline, le contre-poison radical du virus HIV. 70 gouttes, la dose mortelle, deux verres que je fais tourner les yeux fermés. Combien de temps ça prendra pour que mon cœur cesse de battre ? À quoi penserai-je ? À qui ? » (extrait de la bande son du film) Guibert saisit l'un des deux verres et le vide lentement, par petites gorgées. Vient ensuite une mise en suspens introduite par le montage, un plan sur la terrasse vide balayée par le vent, les feuilles d'un journal s'éparpillent au sol tandis que celles des arbres autour se balancent langoureusement au gré des souffles d'airs - retour sur Guibert effondré dans un fauteuil, la tête en arrière, respirant profondément - voix off : "Je suis sorti épuisé de cette expérience, comme modifié, je crois que filmer ça a changé mon rapport à l'idée du suicide. » (extrait de la bande son du film)

Ce qui, au départ, n'était qu'une mise en scène soigneusement préparée finira malgré lui par interagir fortement sur sa vie réelle. De quelle manière l'épreuve filmée du suicide va-t-elle l'influencer, la modifier ? Guibert n'en dira 
pas plus, lui qui finira pourtant par se suicider avec cette même digitaline.

Plus qu'un simple rapport quotidien à la maladie, Guibert touche au plus profond de la dialectique entre pulsion de vie et pulsion de mort - affrontement intense - et véritable mise en «je » du film :

J'étais passé à un autre stade de l'amour de la mort, comme imprégné par elle au plus profond je n'avais plus besoin de son décorum mais d'une intimité plus grande avec elle, je continuais inlassablement de quérir son sentiment, le plus précieux et le plus haïssable d'entre tous, sa peur et sa convoitise (1990a, p. 150).

Chaque jour, la mort gagne du terrain et, pourtant, la vie lui résiste encore, y compris dans les situations les plus désespérées. Cette longue séance de massage, plusieurs heures durant, lutte du corps et des muscles contre l'atrophie qui ronge lentement les chairs, pourrait paraître bien dérisoire si elle n'était pas mue, comme par une force souterraine, par le désir intense de mieux-être, voire de plaisir. Car Guibert continue à désirer, désir qui le fait se mouvoir devant la caméra pour danser presque miraculeusement, désir qui lui donne la force de partir une fois encore sur l'île d'Elbe pour y retrouver des sensations et des lieux aimés, désir d'immersion dans la mer et, par dessus tout, désir d'écrire : «C'est quand j'écris que je suis le plus vivant. Les mots sont beaux, les mots sont justes, les mots sont victorieux [...]. » (1991a, p. 124)

Comme une éclipse de bonheur au milieu du film, l'île d'Elbe, au presbytère de Santa Catarina dans la propriété de son ami Hans Georg Berger, où Guibert avait l'habitude de se rendre. Se rattacher avidement aux formes les plus ténues de bonheur. En voix off dans le film, ces fragments extraits de son 
journal : «Moments exquis de pure jouissance de vie [à l'image, on voit la main de Guibert en train d'ouvrir son cahier noir de travail ], écouter le vent dans les branches, lire quelques lignes de Mémoires d'une naine, puis reposer le livre, rêvasser à ses travaux en cours, observer un lézard perché sur la pomme dans laquelle j'ai croqué hier soir, faire quelques plans en vidéo de contemplation, attendre T. et C. qui vont revenir du marché avec plein de nourritures abondantes, prendre une douche fraîche au soleil, mettre une chemise propre, apaiser sa faim, tout est délice. » (2001, p. 532). Simultanément, un zoom sur la table de jardin sur laquelle est posé le livre, un plan fixe du lézard sur la pomme, un plan de la terrasse avec panoramique droit sur les plantes puis sur un panier rempli de fruits et la table dressée pour trois personnes. La caméra posée sur la table: plan fixe de Guibert mangeant un œuf à la coque et ensuite une pêche - brève incision dans le tableau du bonheur - , la déglutition difficile de Guibert nous ramène à la réalité de sa maladie, puis de nouveau la légèreté Guibert fredonne un petit air joyeux, ses pieds nus sur la table entourée de livres, des plans d'objets familiers, l'arrosoir entre les feuilles d'un arbuste, une faucille, un chapeau en paille, une cisaille, un pot de fleur en terre cuite, le reflet-clin d'œil du soleil dans le rétroviseur d'un vélomoteur. Extraordinaire force vitale, la vie du monde reprend le dessus, déborde du cadre, comme happée par l'énergie qui irradie ces images d'une éternité solaire surexposition - jeux d'ombres et de lumière sur le mur de pierre comme un jeu de cache-cache entre la vie et la mort. À travers ces paysages sonores et colorés, ces objets familiers, Guibert tente de saisir chaque étincelle de vie qui s'offre encore à lui. Filmer participe pleinement de ce désir, désir de retenir par l'image ces quelques derniers instants fugitifs de bonheur. 
Quelles sont les interactions produites par le film dans la vie de l'auteur? Dans quelle mesure l'acte de filmer va-t-il lui permettre de faire émerger des rapports inédits à lui-même, aux autres, au monde qui l'entoure? C'est peut-être à cet instant précis que le film existe réellement. Guibert s'exprime à plusieurs reprises à ce sujet, soit directement dans le film, soit dans ses écrits. La scène du vrai-faux suicide, par exemple, a été totalement inventée au tournage. Sans ce prétexte de mise en scène, Guibert ne l'aurait pas vécue et, pourtant, elle a provoqué chez lui des effets inattendus, au point, nous livre-t-il en voix off, de modifier complètement son rapport à l'idée du suicide : «Le film a opéré la transformation, peut-être comme une catharsis.» (2001, p.532), écrira-t-il dans son journal en décrivant la scène. Comme pour mieux s'en extraire, Guibert se joue de la mort. Roulette russe. Il lui lance un défi. Devant la caméra, il est pris à son propre piège ; la sensation de vertige était bien réelle, imprévisible. Une fois de plus, la frontière entre réalité et fiction s'estompe et l'expérience du film va le démontrer. Un peu comme dans cette citation de Lawrence Durrell chère au cinéaste Jean Daniel Pollet : « Et c'est alors que la réalité première vint au secours de la fiction et que l'imprévisible eut lieu. » (Leblanc et Pollet, p. 18)

Comme Guibert l'exprimait déjà au sujet de l'écriture, le dispositif filmique mis en place va laisser le champ libre aux aléas du réel, à l'imprévisible. « La vidéo s'est révélée un outil complètement approprié. » (1992b, p. 154) avec une caméra relativement légère, accessible, qu'on peut emporter avec soi au cas où le désir de filmer se manifesterait. L'acte de filmer n'est plus séparé de la vie, mais compris dans son mouvement même, sans programmation quelconque. Guibert décide d'emmener sa caméra avec lui à l'hôpital avant une opération: "J'avais 
emporté la caméra, dans un sac FNAC, je me suis dit: "On ne sait jamais, peut-être que je pourrai filmer, peut-être que je n'aurai pas envie, peut-être c'est grotesque, peut-être c'est intéressant, je ne sais pas." » (1992b, p. 151) Le dispositif tout entier s'ouvre à l'imprévisible, au désir ou non de filmer ; les lieux de tournage sont avant tout des lieux de vie : les frontières deviennent poreuses. De même que toute sa trajectoire littéraire entrecroise sans cesse éléments réels et fictifs, sa démarche documentaire intègre ouvertement des aspects fictionnels : le «jeu» du suicide soigneusement mis en scène finira par interagir fortement avec sa vie réelle, presque malgré lui, en faisant émerger des sensations et des émotions inconnues jusqu'alors. Nous touchons ici au cœur de l'interaction vie/cinéma : l'œuvre ne se substitue pas seulement à la vie, elle la transforme. Pour Guibert, il ne s'agit plus uniquement de représenter tel ou tel aspect de sa vie, mais de se mettre en jeu identitairement, de faire naître par les moyens du cinéma des processus psychiques qui n'auraient pas eu lieu sans lui. En acceptant cette prise de risque, Guibert va rendre le film authentiquement parlant et vivant. «Avec la vidéo on s'approche d'un autre instant, un instant nouveau » (2001, p. 531), écrira-t-il à propos de cette scène qui, une fois encore, lui fera découvrir des aspects insoupçonnés de lui-même. Avec l'accord de l'équipe médicale, Guibert filme l'intervention chirurgicale sous anesthésie locale qu'il est en train de subir : il s'agit d'extraire un ganglion à la gorge. Il se filmera ensuite pendant le visionnement de la scène :

J'ai réalisé en voyant le film ce qu'on m'avait fait pour de vrai. Cette lumière chaude irréelle bleue et sableuse à la fois transforme ce qu'elle touche à savoir le champ opératoire : ce trou dans la gorge où affluait et parfois débordait le sang par pulsation en source de lumière incandescente qui censurait le 
sang, y jette des rayons comme à partir d'un noyau radioactif qui est la blessure. (extrait de la bande son du film)

La vidéo va créer cet «instant nouveau » transfiguré par la prise de vue. Guibert revit son opération avec une tout autre intensité, fasciné par l'esthétique supranaturelle de la scène. Les moyens du cinéma auront permis à Guibert de donner une seconde vie à cet instant, de percevoir des sensations nouvelles, des angles de vue inédits.

La présence de la caméra aura également des effets inattendus sur les personnes filmées, comme c'est le cas par exemple avec sa grand-tante Louise, qui s'adresse à lui de manière tout à fait inédite :

Elle proteste quand je parle de suicide, elle me dit : «Ah non, je serais vraiment désolée si tu te suicidais, je serais trop triste, je t'aime figure-toi. »

Elle ne me l'a jamais dit, mais l'intercession de la caméra qui tourne lui permet de le dire c'est incroyable. (1991a, p. 102).

Guibert ne cessera de s'émerveiller et de s'étonner tout à la fois des effets produits dans sa vie quotidienne par l'acte de filmer. Il a pris visiblement beaucoup de plaisir à vivre cette expérience de filmage comme il en aurait sans doute pris pour le montage, si son état physique ne l'avait pas privé de cette étape importante de création.

\section{Interactions film/spectateur}

Interactions vie/cinéma, filmeur/filmé, mais aussi, bien sûr, filmeur/film/spectateur. Comment Guibert s'adresse-t-il au spectateur à travers son film? 
La Pudeur ou l'Impudeur, c'est justement la question posée au spectateur en forme d'interpellation. Le titre initial était «la pudeur et l'impudeur», Le et sera transformé par la suite en ou (voir Artières et Cugnon). Par ce détail qui semble a priori anecdotique, Guibert exprime d'emblée son désir de rencontre avec le spectateur, laissant à celui-ci le champ libre et l'ouverture suffisante pour l'interprétation. Il tient à partager cette expérience du quotidien le plus directement possible, sans intermédiaire, sans le regard extérieur d'un opérateur qui aurait inévitablement introduit une dimension exhibitionniste et voyeuriste au film. Ce dispositif d'auto-filmage, et lui seul, va permettre une telle proximité avec le spectateur — authentique mise en jeu, implication totale du cinéaste. Il est seul face à la caméra comme il est seul face à la mort, et c'est cette expérience extrême qu'il veut partager. À travers sa propre relation spéculaire, Guibert tend le miroir au spectateur, chacun étant renvoyé à lui-même, à son propre rapport à la maladie, à la mort, au suicide.

"Créer un espace où l'expérience puisse se partager », commentait Robert Kramer (p. 8) à propos de ses films. Ce cinéma-là croise avec intensité des «enjeux de vie avec des enjeux de représentation » (Leblanc, 2011). Il ne s'agit pas de détacher la création de la vie, de se substituer à elle mais au contraire d'interagir avec elle. Le film construit des interactions avec la vie du filmeur et avec les personnes filmées en décloisonnant, en brouillant les frontières, en intégrant l'activité artistique aux autres aspects de la vie. De la même manière, le film vise à construire des interactions fortes avec le spectateur. Il ne s'agit pas d'assister au spectacle de la mort au travail, de la déchéance physique de manière voyeuriste. Il ne s'agit pas non plus d'éprouver de la compassion par effet 
d'identification. Le film de Guibert conduit au contraire le spectateur vers une exploration intime de sa propre relation à la vie à travers sa finitude. Pas de manipulation obscène mais un rapport toujours frontal avec le spectateur: Guibert nous regarde dans les yeux, comme il le fait lui-même face à son miroir pour regarder la mort en face. Le spectateur est interpellé à travers la construction d'une relation d'altérité profonde. Une mise en jeu en appelle une autre en retour. Les réactions polémiques qui eurent lieu autour du film font état de deux positions antagonistes d'acceptation ou non de la mise en jeu identitaire par le spectateur.

Pour certains, Guibert va trop loin. Son impudeur est condamnée: on ne peut pas tout montrer, l'image a ses interdits, elle dévoile de façon trop abrupte, on ne peut pas jouer impunément avec la représentation du réel. Pour d'autres, elle dépasse l'intime, le singulier, pour rejoindre l'universel, le partageable.

\section{Bibliographie}

Artières, Philippe et Gilles Cugnon. (2000), La Pudeur ou l'Impudeur d'Hervé Guibert. Genèse d'« un des documentaires les plus bizarres ".

Genon, Arnaud. (2005), «La Pudeur ou l'Impudeur d'Hervé Guibert: l'accomplissement par l'image du dévoilement de soi », dans Lila Ibrahim-Lamrous et Séveryne Muller (dir.), L'Intimité, Clermont-Ferrand, Presses universitaires Blaise Pascal, p. 81-90. 
GuiBerT, Hervé. (1977 [1991]), La Mort propagande, Paris, Régine Deforges.

—. (1988), «Les aveux permanents d'Hervé Guibert », entretien avec Antoine de Gaudemar, Libération, 20 octobre.

—. (1990a), À l'ami qui ne m'a pas sauvé la vie, Paris, Gallimard.

—. (1990b), "La maladie d'amour», entretien avec Antoine de Gaudemar, Libération, $1^{\mathrm{er}}$ mars.

- (1991a), Le Protocole compassionnel, Paris, Gallimard.

—. (1991b), "Hervé Guibert: "J'ai l'impression de survivre" », entretien avec Jérôme Garcin, L'Événement $d u$ jeudi, 26 septembre.

—. (1992), Cytomégalovirus, Paris, Seuil.

-. (1992b), "Pour répondre à quelques questions qui se posent », entretien avec Christophe Donner, La Règle du jeu, $\mathrm{n}^{\circ} 7$, mai.

—. (2001), Le Mausolée des amants, Paris, Gallimard, coll. « Folio ».

-. (2009), La Pudeur ou l'Impudeur, BQHL, 62 minutes, DVD.

Kramer, Robert, Vincent VATRICAN et Cédric Venail (dir.) (2001), Trajets à travers le cinéma de Robert Kramer, Aix-enProvence, Institut de l'image, coll. « Polimago ».

LeBLANC, Gérard. (2003), «Un cinéma du subjectif», Cahier Louis Lumière, no 1, p. 109-125.

—. (2011), «De l'expression subjective directe », Cahier Louis Lumière, no 8, p. 7-17.

- et Jean-Daniel Pollet. (1998), L'entreVues, Montreuil, Éditions de l'œil. 


\title{
Résumé
}

Si l'autobiographie en littérature ou l'autoportait en peinture et en photographie ont été couramment pratiqués, le cinéma semblait moins adapté techniquement à ce type de réalisation intimiste. L'apparition progressive de procédés de filmage plus légers donnera finalement aux cinéastes toute la liberté nécessaire pour l'auto-filmage. Le film d'Hervé Guibert La Pudeur ou l'Impudeur (1991), en utilisant ce type de dispositif, entrecroise des enjeux de vie avec des enjeux de représentation à travers ce qu'on pourrait nommer « un cinéma du subjectif». Un cinéma qui ne serait plus séparé de la vie mais en constante interaction avec elle, ouvert à l'imprévisible, où la mise en jeu identitaire du cinéaste appelle en retour celle du spectateur. Guibert nous regarde dans les yeux, comme il le fait lui-même face à son miroir pour regarder la mort en face.

\begin{abstract}
If autobiography in literature or the self-portrait in painting and photography have long been practiced, film seemed to be technically less suited to a certain type of intimate portrayal. The appearance of lighter filming tools, however, finally brought filmmakers the necessary freedom to « self-film ». In using these new technical devices, Hervé Guibert's film $\mathrm{La}$ Pudeur ou l'Impudeur (1991) crosses life challenges with representational ones through what could be called "a cinema of the subjectivity". This cinema would no longer be separated from life but in constant interaction with life, opened to the unpredictable, in a process which questions the identity game of the filmmaker as well as the one of the viewer. Guibert looks at us straight into the eyes, as he does look straight into his mirror in order to face death.
\end{abstract}

